\title{
Focus of Regulatory Concerns Regarding Pharmacometric Review in the Development and Evaluation of Antimicrobial Agents
}

\author{
Dong Yang ${ }^{1}$, Ming Zhao ${ }^{2}$, Cheng $\mathrm{Lyu}^{3}$, Xingchen $\mathrm{Bian}^{4},{\mathrm{Xin} \mathrm{Li}^{4} \text {, Weiyi Qiu }}^{5}$, Zhiwei \\ Huang ${ }^{4}$, Yuancheng $\mathrm{Chen}^{6}$, Zijian $\mathrm{Hu}^{7}$, and Jing Zhang ${ }^{4}$ \\ ${ }^{1}$ Covance Pharmaceutical Research and Development (Beijing) Co. Limited \\ ${ }^{2}$ China Food and Drug Administration \\ ${ }^{3}$ Shanghai LianBio Development Co., Ltd. \\ ${ }^{4}$ Fudan University Huashan Hospital Institute of Antibiotics \\ ${ }^{5}$ Beijing Institute of Biotechnology \\ ${ }^{6}$ Huashan Hospital Fudan University \\ ${ }^{7}$ CSPC Zhongqi Pharmaceutical Technology (Shijiazhuang) Co., Ltd.
}

August 23, 2020

\begin{abstract}
Pharmacometrics is an emerging science that interprets drug, disease and trial information in a mathematical fashion to inform and facilitate efficient drug development and/or regulatory decisions. Pharmacometric study is increasingly adopted in the regulatory review of new antimicrobial agents. We summarized and reviewed the 31 antimicrobial agents approved by the US Food and Drug Administration (FDA) and the 26 antimicrobial agents approved by European Medicines Agency (EMA) from January 2001 to May 2019. Regulatory concerns in pharmacometric review of antimicrobial agents were expounded with examples, including modeling and simulation methods, effects of internal/external factors on PK parameters, safety and efficacy evaluation in terms of exposure-response analysis, refinement of the wording of product labeling and package leaflet, and possible post-marketing clinical trial. Ongoing communication among regulators, academia, and industry regarding pharmacometrics is encouraged to streamline and facilitate the development of new antimicrobial agents. The industry can maximize its benefit in drug development through continued pharmacometric education/training.
\end{abstract}

\section{BACKGROUND}

Pharmacometrics is an emerging science that pursues a model-based study on the data of pharmacokinetics (PK), pharmacodynamics (PD), body functions, disease processes, and trial project progression using modeling and simulation methods. This emerging discipline usually describes and reflects the relationships between drug dosage, exposure, response, and patient characteristics in terms of concentration-effect, dose-response, and PK-PD correlations. Adoption of pharmacometric approaches at an early stage and in the full course of new drug research and development (R\&D) is helpful for building a model-guided drug R\&D strategy, which is more time- and cost-efficient to shorten drug development course. Therefore, pharmacometrics plays an extremely important role in drug $\mathrm{R} \& \mathrm{D}$, treatment guidance, and regulatory decisions [1-3].

The goal of antimicrobial treatment is to cure patients by inhibiting or killing the invading pathogens and minimize the emergence of bacterial resistance by formulating an effective, safe, and rational dosing regimen. Pharmacometric modeling and simulation are apparently practical tools for the development of antimicrobial agents to achieve such a goal. Pharmacometrics is particularly instrumental in efficacy evaluation, dose selection, regimen optimization, and endpoint analysis when combining the in vitro susceptibility results with the findings of bacterial resistance surveillance. For example, pharmacometric methods can be used to 
define the PK-PD indices and breakpoints indicating antimicrobial activity/susceptibility in clinical patient population by combining PK with the preclinical in vitro susceptibility results; develop the optimal effective and safe dosing regimens for general and special patient populations (e.g., pediatric/geriatric patients, patients with hepatic or renal impairment); and further optimize the dosing regimen in clinical development and post-marketing evaluation $[4,5]$.

Unlike other drug classes, antimicrobial treatment is based on the key mechanism of action of each antimicrobial agent against pathogens. Appropriate PK-PD targets can be determined by associating the microbiological and clinical efficacy with PK-PD indices, such as the percentage of time the free concentration of antibiotic is above the MIC (fT\%>MIC), the ratio between the area under the plasma drug concentration-time curve (AUC) and MIC (fAUC/MIC), and the maximum plasma concentration (Cmax) to MIC ratio (fCmax/MIC). Furthermore, Monte Carlo simulation can be applied to calculate the probability of target attainment (PTA) of different dosing regimens for the selection of optimal dosage and regimen [6, 7]. Pharmacometric researches based on the understanding of tissue penetration can reveal the relationship between the concentration of antimicrobial agent at the site of infection and treatment efficacy, which is more precise than plasma concentration in predicting the antimicrobial efficacy [8].

As members of International Council for Harmonisation of Technical Requirements for Pharmaceuticals for Human Use (ICH), China National Medical Products Administration (NMPA), US Food and Drug Administration (FDA), and European Medicines Agency (EMA) all adopt the important ICH guidelines regarding pharmacometrics, including ICH E1: The Extent of Population Exposure to Assess Clinical Safety; ICH E4: Dose-Response Information to Support Drug Registration; and ICH E5: Ethnic Factors in the Acceptability of Foreign Clinical Data [9]. In addition, regulatory agencies also issued a series of guidelines concerning pharmacometrics according to national or regional reality. These efforts not only reflect the concerns of regulatory authorities but also provide specifications and guidance for the development of antimicrobial agents.

FDA emphasizes the role of pharmacometrics in drug R\&D and review and has rich experience in this respect. It has issued several important guidelines regarding pharmacometrics, e.g., Guidance for Industry: Population Pharmacokinetics (1999), Guidance for Industry: Exposure-Response Relationship - Study Design, Data Analysis, and Regulatory Applications (2003) [10, 11]. The updated version of Guidance for Industry: Population Pharmacokinetics was recently posted online in July 2019 [12]. This document aims to guide the population PK modeling analysis in drug development and new drug application (NDA). The EMA also published a series of pharmacometrics-related guidelines, including Guideline on Reporting the Results of Population Pharmacokinetic Analyses (2007) [13], Guideline on the Use of Pharmacokinetics and Pharmacodynamics in the Development of Antimicrobial Medicinal Products (2016) [14], Guideline on the Evaluation of Medicinal Products Indicated for Treatment of Bacterial Infections (2018) [15], Guideline on the Evaluation of the Pharmacokinetics of Medicinal Products in Patients with Impaired Hepatic Function (CPMP/EWP/2339/02) (2005) [16], and Note for Guidance on the Evaluation of the Pharmacokinetics of Medicinal Products in Patients with Impaired Renal Function (CHMP/EWP/225/02) (2004) [17].

Most of the pharmaceutical products made in China were generic drugs in the past decades. Pharmacometrics is introduced into drug $R \& D$ in China lately. However, in recent years, Chinese regulatory authority has made strong moves to encourage the development of innovative drugs, and facilitate the application of pharmacometrics in new drug development [18]. NMPA has released several guidelines relevant to pharmacometrics, including Technical Guidance on the Pharmacokinetic and Pharmacodynamic Study of Antimicrobial Agents (2017), Technical Guidance on Studying the Breakpoints of Antimicrobial Agents (2018), Technical Guidance on Pharmacokinetic Study in Patients with Impaired Renal Function (2012), Technical Guidance on Pharmacokinetic Study in Patients with Impaired Hepatic Function (2012), and Technical Guidance on Pharmacokinetic Study in Pediatric Patients (2014) [3]. These guidelines provide important specifications and guidance to pharmaceutical companies regarding drug development and evaluation in China. In 1979, the Society of Pharmacometrics under Chinese Pharmacological Society was founded, led by Sun Ruiyuan and Jin Zhengjun. Sun Ruiyuan published the first textbook of Pharmacometrics in China in 1987 [19]. Xie 
Haitang et al compiled an anthology titled Pharmacometrics and New Drug Evaluation [20] in October 2011 on the basis of the proceedings of the first International Conference of Pharmacometrics and New Drug Evaluation which was held in October 2007. This publication summarizes and elaborates on the basic principles of pharmacometrics and its applications in new drug development. Pharmacometrics meetings of Chinese Pharmacological Society and the "International Conference of Pharmacometrics and New Drug Evaluation" have promoted academic communication in the field. The Society of Infectious Diseases under China Medical Education Association published the Expert Consensus on Clinical Application of Antimicrobial Pharmacokinetic/Pharmacodynamic Theory in 2018 [21], which emphasizes the importance of PK-PD in guiding antimicrobial therapy in clinical practice and summarizes the antibacterial and antifungal agents in terms of PK-PD profile. This Consensus document also proposes recommendations and common understanding for optimizing the dosing regimen for common infectious diseases in the patients complicated with chronic organ dysfunction, hypoproteinemia, or infected with resistant microorganism. Liu D., et al published The value and general consideration of pharmacometric study in new drug development in September 2018 [3], which expounds the value, characteristics, application, and technical specifications of pharmacometric study in new drug development and provides insights for new drug R\&D in China.

The drug review reports of China NMPA are not open to the public. So, we here summarize the focus of regulatory concerns in the perspective of pharmacometrics by analyzing the review reports of the antimicrobial agents approved by FDA and EMA from January 2001 to May 2019. We also compared the role of pharmacometric content in FDA-approved and EMA-approved drug labeling and package leaflet for our colleagues' consideration and reference to accelerate and standardize the development of new antimicrobial agents for benefits of patients.

\section{THE ANTIMICROBIAL AGENTS APPROVED BY FDA AND EMA}

The Drugs@FDA database and EMA official website were searched to retrieve the review reports of the antimicrobial agents approved during the period from January 2001 to May 2019. FDA approved 31 antimicrobial agents (Supplementary Table S1), including antibacterial agents (21, 68\%), antifungal agents (9, $29 \%$ ), and antituberculosis drug $(1,3 \%)$ (Table 1, Figure 1). The antibacterial agents (18 single-component and 3 combination products) are licensed to treat community- or hospital-acquired pneumonia, urinary tract infections, skin and skin structure infections. All the approved antifungal agents $(n=9)$ and antituberculosis drug $(\mathrm{n}=1)$ are single-component products. EMA approved 26 antimicrobial agents (Supplementary Table S1), including 18 (69\%) antibacterial agents, 5 (19\%) antifungal agents and $3(12 \%)$ antituberculosis drugs (Table 1, Figure 1). The antibacterial agents (16 single-component and 2 combination products) are approved to treat pneumonia, urinary tract infections, skin, and other infections. All the approved antifungal agents $(\mathrm{n}=5)$ and antituberculosis drugs $(\mathrm{n}=3)$ are single-component products. Overall, 13 antimicrobial agents (9 antibacterial, 3 antifungal, and 1 antituberculous drugs) were approved by both FDA and EMA.

TABLE 1 \& FIGURE 1 PLACEHOLDER

\section{FOCUS OF REGULATORY CONCERNS IN PHARMACOMETRIC REVIEW OF ANTIMI- CROBIAL AGENTS}

From the review reports of the antimicrobial agents approved by FDA and EMA, we summarized the key common concerns of both regulatory agencies in pharmacometric review as follows:

\section{Highly appreciate the use of modeling and simulation methods}

FDA published a white paper titled Challenge and Opportunity on the Critical Path to New Medical Products in 2004, which emphasizes the importance of model-based drug development in new drug R\&D [22]. More than half $(54.8 \%)$ of the 31 FDA-approved antimicrobial agents are supported by population PK modeling analysis (Figure 2 ) for the safety and efficacy evaluation in the NDAs of 12 antibacterial agents, 4 antifungal agents, and 1 antituberculosis drug (including 14 single-component and 3 combination products). Most (73.1\%) of the 26 EMA-approved antimicrobial agents are supported by population PK modeling analysis (Figure 2 ) for the safety and efficacy evaluation in the NDAs of 14 antibacterial agents, 3 antifungal agents, 
and 2 antituberculosis drugs (including 17 single-component and 2 combination products).

Population PK modeling analysis is not available for some drugs, e.g., ozenoxacin (Xepi), efinaconazole (Jublia), and finafloxacin (Xtoro), due to the low-level absorption or lack of systemic exposure after topical administration [23-25]. As for the other drugs without population PK modeling analysis, PK analysis in special populations (in terms of sex, age, hepatic/renal impairment) was provided to support the submission and evaluation of NDA.

Population PK model is built primarily upon the pooled data from phase 1 to 3 clinical trials in healthy subjects and the patients with target indication, which are provided by the applicant. The PK data from animals were also used to build models in some cases. As for combination products (e.g., ceftolozanetazobactam), population PK model should be built for each drug component on a case-by-case basis [26]. If there are major active metabolites, population PK analysis should be conducted for such metabolites. If there are multiple routes of administration (e.g., delafloxacin), multiple models should also be built on a case-by-case basis. In the case of delafloxacin, population PK modeling analysis was provided not only for intravenous administration but also for oral administration [27].

Population PK models are used widely in the evaluation of various antimicrobial agents. Furthermore, Monte Carlo simulation and other tools are also used simultaneously in the PK-PD analysis of antibacterial agents. The MICs of an antibacterial agent against various target pathogens are combined with the PK data to calculate the PTA for the proposed PK-PD target value. Based on the calculated PTA, the probability of different dosing regimens to reach the expected maximalin vivo bactericidal or bacteriostatic effect is evaluated. In this way, the optimal dosing regimen can be recommended with sound rationale. Just take ceftolozane-tazobactam for example, its PTA of PK-PD target was calculated via population PK modeling analysis of ceftolozane alone and tazobactam alone, non-clinical PK-PD target values, in vitro susceptibility testing results, and Monte Carlo simulation [26]. Model selection and development may have a substantial impact on the subsequent simulation. There are advantages and disadvantages for different models (e.g. MIC-based model, killing curve model, and other sophisticated methods), and we should select the appropriate models with justification based on the characters of the compound. In the model development, attention should also be paid to whether it is a concentration-dependent or time-dependent pattern of bactericidal activity observed in time-kill studies, and then select the relevant parameters to predict of efficacy in PK-PD model. Adequacy of the reasons and justification of the extent are essentially important in model building. NONMEM and $\mathrm{R}$ are the software commonly used for modelling. The applicant is required to set up the parameters according to specific data characteristics. For example, a three-compartment model featuring zero-order absorption and first-order elimination was used to describe the plasma concentration-time data pooled from phase 1, 2 and 3 clinical trials of plazomicin. Model selection can be informed by goodness-of-fit plots, including the accuracy of parameter estimates, scatter plot, correlation, and convergence between parameter estimates, modification and conditions of the object function. Non-parametric bootstrapping methods are also appropriate for evaluating the final model and estimating the index with standard deviation and 95\% confidence interval (CI) of population PK parameters [28]. The regulators would repeat the population PK analysis submitted by the applicant to assess its rationality and adequacy in describing the PK data of the drug and the effects of relevant covariates on PK parameters. If any fault is identified, the regulator would correct it, e.g., point out that the parameters and range of sample selection in the model of meropenem-vaborbactam were not set up appropriately by the applicant, and propose to run an independent population PK analysis of updated dataset to assist evaluation.

\section{FIGURE 2 PLACEHOLDER}

\section{Concerns regarding the effects of internal/external factors on PK parameters}

ICH E5 guideline summarizes the possible internal/external factors (covariates) affecting PK parameters. This is also the focus of FDA and EMA concerns in pharmacometric review. The common internal factors include race, genetic polymorphisms, sex, age, body weight, body mass index, and hepatic/renal functions. The frequently mentioned external factors are diet, treatment compliance, treatment modality, and infectious 
pathogen [9]. Correct selection of covariates is very important, which should be based on the specific characteristics and dosing regimen of a drug. Ceftazidime-avibactam (Avycaz), for example, is eliminated through kidneys, so parameters of renal function like augmented renal clearance and end-stage renal disease (ESRD) are among the significant covariates [29]. Race is a significant covariate of isavuconazole because the plasma level of isavuconazole is lower in westerners than in Chinese descents [30]. In the case of mixed infection caused by multiple pathogens, it is required to analyze the effect of these pathogens on PK parameters.

The interaction between the active ingredients of a combination of products is also a focus of concern. For instance, vabomere is a combination of meropenem and vaborbactam. So, it is required to assess the effect of vaborbactam on the PK profile of meropenem [31]. In addition to ingredient interaction analysis, it is also of crucial importance to investigate the possibility of drug-drug interactions (DDIs). For example, the data from the in vitro and in vivo studies of ceftazidime-avibactam combination were used to investigate whether there was any significant interaction between drug ingredients and the substrates, inhibitors, and inducers of cytochrome P450 enzymes.

Covariates analysis may be affected by trial design and implementation. For example, more than $60 \%$ of the administered dose of ceftaroline fosamil is eliminated in urine in unchanged form. However, urinary excretion data of ceftaroline were not available in phase $2 / 3$ clinical trials. It was impossible to assess the significant covariate (i.e., renal clearance) of ceftaroline simply based on population PK model [32]. This is also the case of antibacterial agent dalbavancin [33].

\section{Concerns regardingexposure-response relationship for efficacy evaluation}

Exposure-response analysis a very useful method for evaluating the efficacy of drugs and therefore a focus of FDA and EMA concern in regulatory review. Except the topical agents lacking systemic exposure, all antimicrobial agents are evaluated through exposure-response analysis to determine whether the dosage and efficacy outcome data from the completed clinical trials are adequate to support its efficacy in the proposed indications. Vabomere (meropenem-vaborbactam) is an example in this case. Population PK models, nonclinical PK-PD analysis and Monte Carlo simulation are used to calculate the probability of achieving PK-PD target. These results are combined with in vitrosusceptibility data to predict the clinical efficacy of the drug. All the available efficacy endpoints of oritavancin in the patients with acute skin and soft tissue infection caused by $S$. aureus were used to establish PK-PD correlation for evaluating the efficacy of oritavancin in patients with $S$. aureus infection [34].

When sufficient data of PK, PD and microbiology tests are available from clinical trials, it is appropriate to conduct analyses of dose exposure-response to determine the highest MIC permissible for clinical efficacy of a dosing regimen, clinical PK-PD target value, and the PTA at a specific MIC. If the results of clinical trials showed clinical failure in very few cases or low prevalence of target pathogens, it is impossible to establish the quantitative relationship between PK-PD indices and clinical or microbiological efficacy. If clinical PK-PD target value is not available, it is recommended to use the PK-PD target value from animals or in vitro studies and Monte Carlo simulation to evaluate the dosing regimen. It is very important to determine the breakpoints of an antimicrobial agent in exposure-response analysis, which involves multidisciplinary study covering in vitro andin vivo preclinical studies, clinical trials and microbiological testing, and comprehensive analysis. In the example of ceftolozane-tazobactam, clinical efficacy data were not available for the patients at higher MIC. FDA reviewers, therefore, selected a relatively conservative target value (equivalent to $2-\log _{10}$ kill effect) according to the clinical efficacy of ceftolozane-tazobactam in the patients with complicated intra-abdominal infections (cIAI). Accordingly, the susceptibility breakpoint of this drug against Enterobacteriaceae andPseudomonas aeruginosa is $2 \mu \mathrm{g} / \mathrm{mL}$ and $4 \mu \mathrm{g} / \mathrm{mL}$, respectively, lower than the breakpoints $(8 \mu \mathrm{g} / \mathrm{mL}$ for both Enterobacteriaceaeand $P$. aeruginosa ) proposed by the applicant [35]. The exposure-response relationship is evaluated more reasonably by this breakpoint modification [34].

\section{Concerns regarding exposure-response relationship for safety evaluation}

Exposure-response analysis is also a focus of FDA and EMA concerns for safety evaluation. All antimicrobial agents should be evaluated for their safety profile in general and special populations by analyzing 
the correlation between dose/exposure and the safety endpoints and adverse reactions defined in clinical trials. In the example of ceftaroline fosamil, QT interval prolongation was considered as a safety endpoint in the exposure-response analysis [36]. One more example, voriconazole is metabolized by cytochrome P450 isozymes CYP2C19, CYP2C9 and CYP3A4 and these P450 isozymes are inhibited by voriconazole. So, if patients use the inhibitors or inducers of these isozymes, voriconazole should be prescribed cautiously, especially in the patients receiving concomitant treatment which are associated QT prolongation [37].

\section{Pharmacometric review helps refine the wording of dosing regimen and labeling}

Exposure-response analysis is the key to analyzing the appropriateness of dosing regimen (dosage, frequency, mode and duration of administration) and so the focus of regulatory concern. FDA and EMA will determine whether the proposed dosing regimen is reasonable; whether the recommended dosage is acceptable in general adult population and special populations (e.g., geriatric, pediatric or patients with impaired renal/hepatic function) by examining the exposure-response relationship in terms of drug efficacy and safety. If not, the Agency will decide how to adjust the dosage or dosing regimen. The corresponding method and wording of dose adjustment are also required to be reflected in the package leaflet (labeling). For instance, the exposureresponse analysis in terms of efficacy and safety supports Vabomere $4 \mathrm{~g}$ (meropenem : vaborbactam $=1: 1)$ by intravenous infusion (IV) over 3 hours, every 8 hours (q8h), up to 14 days in adult patients ([?] 18 years old) with adequate renal function evidenced by estimated glomerular filtration rate (eGFR) [?] 50 $\mathrm{mL} / \mathrm{min} / 1.73 \mathrm{~m}^{2}$. However, for patients with impaired renal function (eGFR $<50 \mathrm{~mL} / \mathrm{min} / 1.73 \mathrm{~m}^{2}$ ), the dosing regimen should be adjusted. Specifically, Vabomere $2 \mathrm{~g}$, IV q8h if eGFR ranges from 30 to 49 $\mathrm{mL} / \mathrm{min} / 1.73 \mathrm{~m}^{2}$; Vabomere $2 \mathrm{~g}$ IV q12h if eGFR ranges from 15 to $29 \mathrm{~mL} / \mathrm{min} / 1.73 \mathrm{~m}^{2}$; and Vabomere $1 \mathrm{~g}$ IV $\mathrm{q} 12 \mathrm{~h}$ if eGFR is lower than $15 \mathrm{~mL} / \mathrm{min} / 1.73 \mathrm{~m}^{2}$ [38].

\section{Pharmacometric review may trigger post-marketing clinical trial}

Pharmacometric review also provides important evidence for justifying the adequacy of the completed preclinical and clinical studies in support of NDA. For example, ceftazidime is excreted through kidneys, dose adjustment is required for ceftazidime-avibactam in the patients with renal impairment. Based on pharmacometric review, FDA reviewers advised the applicant to conduct a post-marketing study on ceftazidimeavibactam in cIAI patients whose creatinine clearance [?] $50 \mathrm{~mL} / \mathrm{min}$ to assess the PK, efficacy, and safety after dose adjustment. The PK results from the proposed post-marketing trial are used to analyze whether it is necessary to further adjust the dosing regimen. Another example is the antituberculosis drug delamanid. The relationship between the exposure and antibacterial activity of delamanid was inconsistent. So EMA reviewers request the applicant to perform a post-marketing study to further examine the correlation between 2-month sputum conversion and long-term efficacy following different dosing regimens.

From the relevant NMPA guidelines and the prescribing information of some new antimicrobial agents approved in China, we can see that NMPA has essentially the same focus of concerns as FDA and EMA in pharmacometric review. NMPA approved the new antibacterial agent nemonoxacin malate capsules in 2016. Various PK parameters of nemonoxacin were evaluated thoroughly in the heathy subjects and the patients with community-acquired pneumonia. Population PK analysis was also provided to examine the effect of multiple covariates (sex, age, the severity of renal impairment, and diet) on PK. These analyses provide robust justification of the efficacy and safety of the drug in patients and demonstrate whether it is necessary to adjust dosing regimen [39].

\section{PHARMACOMETRICS INFORMS LABELING AND PACKAGE LEAFLET OF ANTIMI- CROBIAL AGENTS}

The EMA-approved labelling of antimicrobial agents is concise in content, but the package leaflet provides an easy-to-understand summary of product characteristics for patients and healthcare professionals separately, and detailed information about storage, usage, and precautions. However, the data and information relevant to pharmacometrics are infrequently described in EMA-approved labeling and package insert. This is consistent with EMA Guideline on theReadability of the Labelling and Package Leaflet of Medicinal Products for Human Use published in 2009 [40]. 
The FDA-approved labeling of antimicrobial agents has relatively profuse content. The pharmacology section includes the data of drug absorption, distribution, metabolism, and excretion in healthy subjects and patients, including those with impaired hepatic/renal function. The effect of covariates on exposure and population PK analysis (if any) is also described. The results of in vivo and in vitro DDI studies are also summarized in labeling to inform if there is any drug incompatibility or contraindication. FDA also issued a series of guidelines, such as Clinical Studies Section of Labeling for Human Prescription Drug and Biological Products - Content and Format in 2006 and Clinical Pharmacology Labeling for Human Prescription Drug and Biological Products - Content, and Format (2016) [41, 42]. These guidelines provide clear-cut guidance and specifications for the industry regarding the content and format of the pharmacology section of drug product labeling. At the Precision Dosing Public Workshop held on August 12, 2019, Robert Temple from Center for Drug Evaluation and Research (CDER) of FDA also advised FDA to consider adding the content of PK-PD modeling analysis to drug labeling for guiding individualized dosing.

NMPA also highly values the importance of the standardization and management of drug package leaflet and labeling and provides a clear guidance to the industry in relevant policies, includingRegulations for Management of Drug Package Leaflet and Labeling, Detailed Specifications for Package Leaflet of Chemical Drugs and Biological Products, and Technical Guidance for Drafting Package Leaflet of Antimicrobial Agents - We can see that NMPA clearly advises applicants to include the information regarding antimicrobial susceptibility testing, results of PK and PD parameters, DDI study, precautions in special population, and the effect of various covariates on PK in the package leaflet, for better clinical use of the drug by patients and healthcare professionals.

\section{PHARMACOMETRICS INTEGRATED INTO THE R\&D OF ANTIMICROBIAL AGENTS IN CHINA}

Pharmacometrics, as an emerging discipline, started relatively later in China. The application of pharmacometric approaches in new drug development is inadequate in depth and extent. Among the antimicrobial agents receiving regulatory evaluation, only a few drugs such as nemonoxacin have a full set of pharmacometric studies.

Nonetheless, in recent years, China scientists have participated in more and more international multi-center clinical trials. A number of innovation-based pharmaceutical companies are emerging. It is encouraging to note the buy-in of pharmacometric concepts by the industry due to vigorous promotion by the regulator and academia. China has made great progress in new drug R\&D partly thanks to the application of pharmacometrics. However, the knowledge gap and misunderstanding still exist among drug manufacturers about the advantages, role, and application of pharmacometrics in new drug development. Consistently integrating pharmacometric methods into new drug development still requires concerted efforts of the regulator, academia, and industry in China.

\section{TIPS FOR THE INDUSTRY TO LEVERAGE PHARMACOMETRIC REVIEW OF AN- TIMICROBIAL AGENTS}

Pharmacometrics is apparently important in drug R\&D. The manufacturers with an intention to develop antimicrobial agents should take advantage of pharmacometrics to understand the PK-PD profile of the drug under study and build physiologically based PK (PBPK) and population PK models at early stage of drug development. The in vitro susceptibility testing data of clinical pathogenic isolates (bacteria or fungi) can be combined with PK data to inform trial design and the dosing regimens in clinical trials, to identify the optimal dosing regimen, and accelerate new drug launch. As drug R\&D advances, realtime modeling is recommended by dynamic update of the predefined model to better understand which physiological, pathological, concomitant medication or other factors of patients are the significant covariates affecting the PK profile of the drug, and provide adequate data to support drug safety and efficacy. The following measures may be helpful for relevant entities to benefit from pharmacometrics.

- Recruit dedicated pharmacometricians, provide professional training, build specialized pharmacometrician team or assign the pharmacometrics responsibility to a clinical pharmacology team. The research- 
based pharmaceutical companies should make the size of their pharmacometrician team appropriate to their product pipeline.

- Actively encourage the staff members to attend pharmacometric training program. Pharmacometrics is developing rapidly as an emerging discipline with ever-changing research hot spots. The concerned pharmaceutical companies are recommended to keep an eye on the latest training information and develop a customized training plan according to the background knowledge and experience of pharmacometric staff.

- Integrate pharmacometric strategy and study into Company's Target Product Profile (TPP), Clinical Development Plan (CDP), and Pharmacology Development Plan (PDP) to boost product development. Innovative pharmacometric methods should also be considered in the development of generic drugs to conduct modeling analysis of key parameters for bridging the safety and efficacy of the generic drug with the original brand drug, improving the quality of generic drugs, and shortening the development cycle.

- Actively communicate the strategy of pharmacometric study and development milestones with the regulatory agencies for guidance and advice from the regulator.

- Include the data of pharmacometric study in the clinical trial application and NDA package submitted to Chinese regulatory agency and to standardize the wording of package leaflet.

- Actively attend pharmacometric meetings held regionally or globally to communicate scientific findings and advance pharmacometric research.

The pharmaceutical companies should also pay attention to the following issues when applying the pharmacometric strategy and approaches to new drug development:

- The requirement for pharmacometric study may vary with the stage of new drug development and evaluation. Pharmaceutical companies should plan scientifically with reference to current guidelines. During the development of innovative antimicrobial agents, PK data should be combined with in vitro bacteriostatic results. Population PK data should be combined with PK-PD analysis to optimize the dosing regimen. The clinical and microbiological efficacy data should be collected in clinical studies for dynamic pharmacometric analyses.

- At each stage of new drug development, the industry should work with the investigator and regulator to conduct analysis and employ the strategy of pharmacometric study for an individual drug.

- The results of pharmacometric study should be confirmed in subsequent clinical trials or practice.

In conclusion, pharmacometrics is an intricate emerging discipline involving clinical pharmacology, physiology, pharmacology, toxicology, clinical medicine, microbiology, biology, and statistics. Pharmaceutical companies should strengthen pharmacometric team building via systematic professional education and training to meet the requirements of increasingly complicated new drug development.

\section{References}

1. Barrett JS, Fossler MJ, Cadieu KD, Gastonguay MR. Pharmacometrics: a multidisciplinary field to facilitate critical thinking in drug development and translational research settings. J Clin Pharmacol 2008; 48: $632-49$.

2. Ette EI, Williams PJ. Pharmacometrics: the science of quantitative pharmacology: John Wiley \& Sons, 2013.

3. Liu D, Ma G, Xiang X, Liu J, Zhao P, Chen R, Chen Y, Huang X, Li L, Li L, Nie J, Wang Y, Wei C, Lu W, Shi J, Li G, Yang J, Wang Y, Zheng Q, Hu B. The value and general consideration of pharmacometric study in new drug development. Chin J Clin Pharmacol Ther 2018; 23: 961-73.

4. Asín-Prieto E, Rodríguez-Gascón A, Isla A. Applications of the pharmacokinetic/pharmacodynamic (PK/PD) analysis of antimicrobial agents. J Infect Chemother 2015; 21: 319-29.

5. Kitamura Y, Yoshida K, Kusama M, Sugiyama Y. A proposal of a pharmacokinetic/pharmacodynamic (PK/PD) index map for selecting an optimal PK/PD index from conventional indices (AUC/MIC, 
Cmax/MIC, and TAM) for antibiotics. Drug Metab Pharmacokinet 2014; 29: 455-62.

6. Tunkel AR, Scheld. Aztreonam. Infect Control Hosp Epidemiol 1990; 11: 486-94.

7. Zhang J. Clinical Pharmacology of Antimicrobial Agents. In: Practical Anti Infective Therapy, 2013: $87-110$.

8. Rizk M, Zou L, Savic R, Dooley KE. Importance of drug pharmacokinetics at the site of action. Clin Transl Sci 2017; 10: 133-42.

9. ICH. Efficacy Guidelines. <https://www.ich.org/page/efficacy-guidelines>. 2020. Accessed February 17 2020 .

10. FDA. Guidance for industry: population pharmacokinetics. Rockville: US Food and Drug Administration 1999.

11. FDA. Exposure-Response Relationships - Study Design, Data Analysis, and Regulatory Applications. <https://www.fda.gov/regulatory-information/search-fda-guidance-documents/exposure-responserelationships-study-design-data-analysis-and-regulatory-applications>. 2003. Accessed December 12019.

12. FDA. Population Pharmacokinetics. <https://www.fda.gov/regulatory-information/search-fdaguidance-documents/population-pharmacokinetics>. 2019. Accessed February 22020.

13. EMA. Guideline on reporting the results of population pharmacokinetic analyses. <https://www.ema.europa.eu/en/documents/scientific-guideline/guideline-reporting-results-populationpharmacokinetic-analyses_en.pdf>. 2007. Accessed February 162020.

14. EMA. Guideline on the use of pharmacokinetics and pharmacodynamics in the development of antimicrobial medicinal products. <https://www.ema.europa.eu/en/documents/scientific-guideline/guideline-usepharmacokinetics-pharmacodynamics-development-antimicrobial-medicinal-products_en.pdf $>$. 2016. Accessed February 162020.

15. EMA. Guideline on the evaluation of medicinal products indicated for treatment of bacterial infections. <https://www.ema.europa.eu/en/documents/scientific-guideline/draft-guideline-evaluation-medicinalproducts-indicated-treatment-bacterial-infections-revision-3_en.pdf>. 2018. Accessed February 162020.

16. EMA. Guideline on the evaluation of the pharmacokinetics of medicinal products in patients with impaired hepatic function (CPMP/EWP/2339/02). < https://www.ema.europa.eu/en/documents/scientificguideline/guideline-evaluation-pharmacokinetics-medicinal-products-patients-impaired-hepatic-function_en.pdf >. 2005. Accessed February 162020.

17. EMA. Note for guidance on the evaluation of the pharmacokinetics of medicinal products in patients with impaired renal function (CHMP/EWP/225/02). <https://www.ema.europa.eu/en/documents/scientificguideline/note-guidance-evaluation-pharmacokinetics-medical-products-patients-impaired-renal-function_en.pdf >. 2004. Accessed February 152020.

18. Ma G, Xu L, Chen R, Chen Y, Zhao W, Liu D, Jiao Z, Li J, Ji S, Li L, Li L, Wang Y, Yang J, Wang Y, Sun H, Hu B, Zheng Q, Wei L. General considerations of population pharmacokineticpharmacodyna-mic study in new drug development. Chin J Clin Pharmacol Ther 2019; 24: 1201-20.

19. Sun R. Quantitative pharmacology: Beijing: People's Medical Publishing House, 1987.

20. Xie H. Quantitative Pharmacology and Drug Development: People's Military Medical Publishing House, 2011.

21. Zhang J, Lv Y, Yu K, Guan X, Huang Y, Kang Y, Cui J, Liang B, Yang Y, Yan J. Expert Consensus on Antibiotics Pharmacokinetics/Pharmacodynamics Clinical Application. Chin J Tuber and Respir Dis 2018; 41: 409-46. 
22. FDA. Challenge and opportunity on the critical path to new medical products. http://www fda gov/oc/initiatives/criticalpath/ 2004.

23. Vila J, Hebert AA, Torrelo A, López Y, Tato M, García-Castillo M, Cantón R. Ozenoxacin: a review of preclinical and clinical efficacy. Expert Rev Anti Infect Ther 2019; 17: 159-68.

24. Gupta AK, Simpson FC. Efinaconazole (Jublia) for the treatment of onychomycosis. In: Taylor \& Francis, 2014.

25. McKeage K. Finafloxacin: first global approval. Drugs 2015; 75: 687-93.

26. Chandorkar G, Xiao A, Mouksassi MS, Hershberger E, Krishna G. Population pharmacokinetics of ceftolozane/tazobactam in healthy volunteers, subjects with varying degrees of renal function and patients with bacterial infections. J Clin Pharmacol 2015; 55: 230-39.

27. Zhang L, Bhavnani SM, Ambrose PG, Cammarata SK, Rubino CM. Population Pharmacokinetic (PPK) Analysis for Intravenous (IV) and Oral (PO) Delafloxacin Using Data From Phase 1 Studies. Open Forum Infect Dis 2016; 3 .

28. Trang M, Seroogy JD, Van Wart SA, Bhavnani SM, Kim A, Gibbons JA, Ambrose PG, Rubino CM. Population pharmacokinetic analyses for plazomicin using pooled data from phase 1, 2, and 3 clinical studies. Antimicrob Agents Chemother 2019; 63: e02329-18.

29. Li J, Lovern M, Green ML, Chiu J, Zhou D, Comisar C, Xiong Y, Hing J, MacPherson M, Wright JG. Ceftazidime-avibactam population pharmacokinetic modeling and pharmacodynamic target attainment across adult indications and patient subgroups. Clin Trans Sci 2019; 12: 151-63.

30. Desai A, Kovanda L, Kowalski D, Lu Q, Townsend R, Bonate PL. Population pharmacokinetics of isavuconazole from phase 1 and phase 3 (SECURE) trials in adults and target attainment in patients with invasive infections due to Aspergillus and other filamentous fungi. Antimicrob Agents Chemother 2016; 60: 5483-91.

31. Rubino CM, Bhavnani SM, Loutit JS, Morgan EE, White D, Dudley MN, Griffith DC. Phase 1 study of the safety, tolerability, and pharmacokinetics of vaborbactam and meropenem alone and in combination following single and multiple doses in healthy adult subjects. Antimicrob Agents Chemother 2018; 62: e02228-17.

32. Van Wart SA, Forrest A, Khariton T, Rubino CM, Bhavnani SM, Reynolds DK, Riccobene T, Ambrose PG. Population pharmacokinetics of ceftaroline in patients with acute bacterial skin and skin structure infections or community-acquired bacterial pneumonia. J Clin Pharmacol 2013; 53: 1155-67.

33. Buckwalter M, Dowell JA. Population pharmacokinetic analysis of dalbavancin, a novel lipoglycopeptide. J Clin Pharmacol 2005; 45: 1279-87.

34. Bhavnani SM, Passarell JA, Owen JS, Loutit JS, Porter SB, Ambrose PG. Pharmacokineticpharmacodynamic relationships describing the efficacy of oritavancin in patients with Staphylococcus aureus bacteremia. Antimicrob Agents Chemother 2006; 50: 994-1000.

35. Hong M-C, Hsu DI, Bounthavong M. Ceftolozane/tazobactam: a novel antipseudomonal cephalosporin and $\beta$-lactamase-inhibitor combination. Infect Drug Resist 2013; 6: 215-23.

36. Riccobene TA, Rekeda L, Rank D, Llorens L. Evaluation of the effect of a supratherapeutic dose of intravenous ceftaroline fosamil on the corrected QT interval. Antimicrob Agents Chemother 2013; 57: 177783.

37. Theuretzbacher U, Ihle F, Derendorf H. Pharmacokinetic/pharmacodynamic profile of voriconazole. Clin Pharmacokinet 2006; 45: 649-63. 
38. Trang M, Dudley MN, Bhavnani SM. Use of Monte Carlo simulation and considerations for PK-PD targets to support antibacterial dose selection. Curr Opin Pharmacol 2017; 36: 107-13.

39. Guo B, Wu X, Zhang Y, Shi Y, Yu J, Cao G, Zhang J. Safety and clinical pharmacokinetics of nemonoxacin, a novel non-fluorinated quinolone, in healthy Chinese volunteers following single and multiple oral doses. Clin Drug Investig 2012; 32: 475-86.

40. EMA. Guideline on the Readability of the Labelling and Package Leaflet of Medicinal Products for Human Use. In: Revision, 2009.

41. FDA. Clinical Pharmacology Labeling for Human Prescription Drug and Biological Products - Content, and Format. <https://www.fda.gov/regulatory-information/search-fda-guidance-documents/clinicalpharmacology-labeling-human-prescription-drug-and-biological-products-content-and-format $>$. 2016. Accessed February 142020.

42. FDA. Clinical Studies Section of Labeling for Human Prescription Drug and Biological Products - Content and Format. <https://www.fda.gov/regulatory-information/search-fda-guidance-documents/clinicalstudies-section-labeling-human-prescription-drug-and-biological-products-content-and-format $>$. 2006. Accessed February 142020.

Table 1 Summary of the antimicrobial agents approved by FDA and EMA from January 2001 to May 2019

\begin{tabular}{llllll}
\hline Product type & FDA-approved & FDA-approved & FDA-approved & EMA-approved & EMA-approv \\
\hline & Total & Combination & Single-component & Total & Combination \\
Antibacterial/antibiotic & 21 & 3 & 18 & 18 & 2 \\
Antifungal agent & 9 & 0 & 9 & 5 & 0 \\
Anti-tuberculosis drug & 1 & 0 & 1 & 3 & 0 \\
\hline
\end{tabular}

EMA, European Medicines Agency; FDA, US Food and Drug Administration.

Figure Legends

Figure 1. Number, percentage, and types of the antimicrobial agents approved by FDA (A) and EMA (B) from January 2001 to May 2019. EMA, European Medicines Agency; FDA, US Food and Drug Administration.

Figure 2. The antimicrobial agents approved by FDA and EMA from January 2001 to May 2019 in terms of population PK modeling support. Panel A, by type (anti-tuberculosis, antifungal, or antibacterial/antibiotic); Panel B by component (single-component or combination product). EMA, European Medicines Agency; FDA, US Food and Drug Administration; Pop PK, population pharmacokinetic.

\section{Appendices:}

Table S1. List of the antimicrobial agents approved by FDA and EMA from January 2001 to May 2019 
Figure 1

A

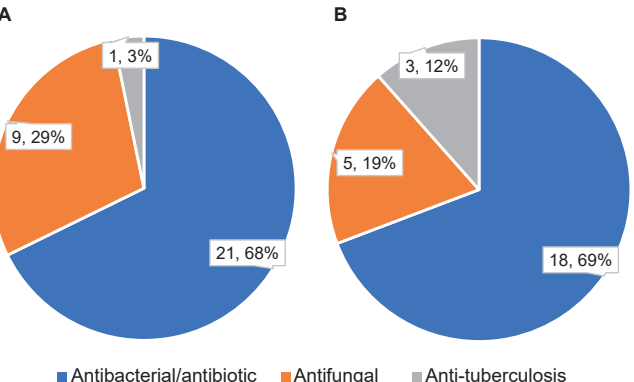




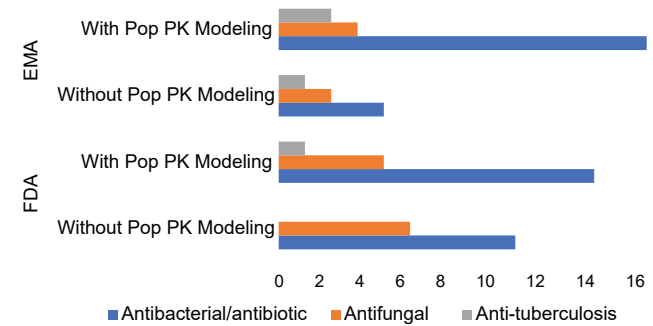

B

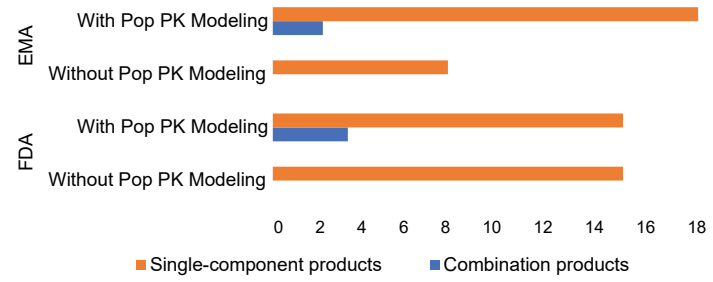

